\title{
Sisal cellulose acetates obtained from heterogeneous reactions
}

\author{
M. Peres de Paula, T. M. Lacerda, E. Frollini* \\ Instituto de Química de São Carlos, Universidade de São Paulo; Av. Trabalhador Sãocarlense - 400, CP 780, \\ 13560-970 - São Carlos, São Paulo, Brazil
}

Received 18 February 2008; accepted in revised form 15 April 2008

\begin{abstract}
In the present work, cellulose obtained from sisal, which is a source of rapid growth, was used. Cellulose acetates were produced in heterogeneous medium, using acetic anhydride as esterifying agent and iodine as catalyst, to check if the procedure described in the literature for commercial cellulose also is adequate to sisal cellulose. The results indicated that iodine is an excellent catalyst to obtain sisal cellulose acetates, but the reaction is so fast as described in the literature when, instead of sisal, lower average molar weight cellulose (microcrystalline) is used. The crystallinity index $\left(I_{c}\right)$ of sisal cellulose acetates diminished compared to sisal cellulose, but there was no direct correlation between their degree of substitution (DS) and $I_{c}$. Probably acetyl groups were introduced more homogeneously along the short chains of microcrystalline cellulose, when compared to sisal cellulose, and then for microcrystalline cellulose acetates the $I_{c}$ decreases as DS increases. Using the linear correlation that was found between degree of substitution (DS) and time reaction is possible to control the DS of sisal cellulose acetates, considering a large interval of degrees of substitution (0.3-2.8).
\end{abstract}

Keywords: biopolymers, sisal cellulose, cellulose acetates, iodine, catalyst

\section{Introduction}

Cellulose can be extracted from several natural sources, as for instance wood, cotton, sisal, sugar cane bagasse and bamboo. In the present study, sisal was used as cellulose source because of its short cultivation period (annual harvest), in contrast to trees from which wood, the main and current source of cellulose, is obtained [1-4]; and also on account of its high cellulose content [2, 3].

The production of cellulose derivatives has extensive interest worldwide, mainly because of its abundance in nature, its biodegradability and its lower environmental impact in comparison with polymers obtained from fossil sources [5]. Among the aspects that demand continuous basic research, the production of cellulose acetate can be cited for its vast industrial application such as textile (cloth- ing and fabrics), high absorbency products (diapers, cigarette filters and other filters), thermoplastics products (films and plastic instruments), nourishing (food packaging), cosmetic and pharmaceutical (extended capsule/tablet release agents and encapsulating agent), medicinal (hypoallergenic surgical products) and others.

Cellulose esterification process basically uses acid anhydrides or acyl chlorides as acetylating agents. Efforts are constantly directed to finding conditions that minimize the time and temperature of reactions and/or lead to better controlling stoichiometry, amid other parameters of reactions. In previous works, our interest addressed the reactions of cellulose in homogeneous medium, involving celluloses from several plant sources (linters, cotton, sisal and 
sugarcane bagasse) $[2-4,6-10]$ mainly aiming at obtaining acetates.

Cellulose derivatives, as esters, are largely industrially produced in heterogeneous medium. Cellulose esterification process basically uses anhydrides acids or acyl chlorides as acetylating agents. Efforts are continually directed to finding conditions that minimize the time and temperature of reactions and/or lead to better controlling the desired stoichiometry, amid other reaction parameters.

The present paper concerns the preparation of cellulose acetates in heterogeneous medium using iodine as catalyst. The procedure was based on that reported by Biswas et al. [11, 12] which is related to acetylation of commercial cellulose, considering short periods of time and using iodine as catalyst. Figure 1 illustrates the mechanism proposed for cellulose acetylation, using iodine as catalyst.

The reaction is supported by the fact that iodine shows great efficiency in catalyzing the esterification of primary and secondary alcohols, as recently demonstrated $[13,14]$. This catalyst is resistant to humidity, with no adverse environmental impact and can be recycled.

The purpose of the present study was to evaluate the influence of cellulose properties, such as the average molar mass and crystallinity on the efficiency of this acetylation reaction. Besides sisal cellulose microcrystalline cellulose was used for comparison reasons.

\section{Experimental}

\subsection{Materials}

The sisal cellulose was generously provided by Lwarcell (Lençóis Paulista, SP, Brazil) and Avicel PH-101 microcrystalline cellulose, by FMC, Inc. (São Paulo, Brazil).

\subsection{Cellulose characterization}

The average degree of polymerization (DP) was determined by viscometry, using an Ostwald viscometer, as described elsewhere [9].

Crystallinity index $\left(I_{c}\right)$ was determined by X-ray diffraction, using a VEB CARL ZEISS-JENA URD-6 Universal Diffractometer operating at $40 \mathrm{kV} / 20 \mathrm{~mA}$ and $\lambda\left(\mathrm{CuK}_{\alpha}\right)=1.5406 \AA$. The crystallinity index was calculated using the BuschleDiller and Zeronian equation, as described elsewhere [15].

\subsection{Cellulose mercerization}

Sisal was mercerized in $20 \% \mathrm{NaOH}$ solution (w/w) at $0^{\circ} \mathrm{C}$ for $1 \mathrm{~h}$, under stirring, as described in previous work [9].

\subsection{Celluloses acetylation}

The procedure was based on that described by Biswas and coworkers [12]. $0.5 \mathrm{~g}$ of cellulose (sisal or microcrystalline) was suspended in $8.5 \mathrm{ml}$ of acetic anhydride in a glass reactor equipped with mechanic stirring, and reflux condenser. The mixture was heated to $100^{\circ} \mathrm{C}$ and then $0.05 \mathrm{~g}$ of iodine was added. At this point, the mixture color turns to dark brown. The intervals of reaction time were varied. At the end of the reaction, the mixture was cooled at room temperature and then $2 \mathrm{ml}$ of saturated solution of sodium thiosulfate was added, in order to neutralize the iodine action. This procedure makes the mixture change to colorless. Thus, $50 \mathrm{ml}$ of ethanol was added and the mixture was stirred for $20 \mathrm{~min}$. The cellulose acetates obtained remained suspended in this medium. Afterward, the mixture was filtered and thoroughly washed with water, dried at room temperature and then at $60^{\circ} \mathrm{C}$ under vacuum, until constant weight.

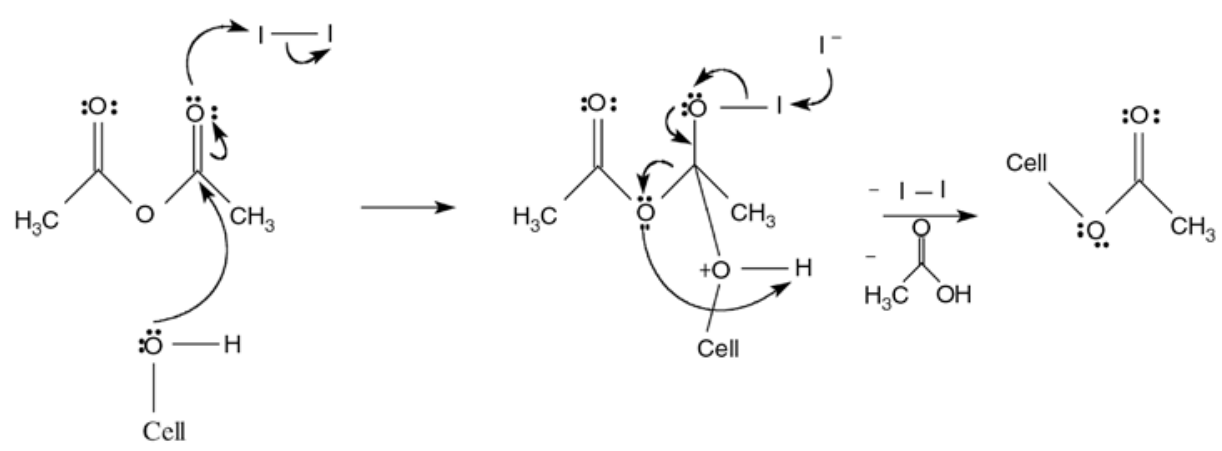

Figure 1. Mechanism of cellulose (Cell-OH) acetylation using iodine as catalyst (based on Biswas et al. [11]) 


\subsection{Cellulose acetates characterization}

Degree of substitution: the degree of substitution (DS) of cellulose acetates was determined by proton NMR, as described elsewhere [2, 7]. Spectra were recovered with a Brucker AC-200 spectrometer running at $200 \mathrm{MHz}$ and $80^{\circ} \mathrm{C}$. The number of scans was about 390. Samples were dissolved in DMSO- $d_{6}$ in $5 \mathrm{~mm}$ diameter NMR tubes $(10 \mathrm{mg} / \mathrm{ml})$. A drop of trifluoracetic acid was added to the sample solution in order to shift the signal of residual water and hydroxyl protons to a lower field outside the spectral region of interest, without affecting the chemical shifts of the glucose ring protons [16].

Infrared spectroscopy: the equipment used was a BOMEM, MB-102 model. The preparation of the pellets was made with $1 \mathrm{mg}$ of the sample added to $100 \mathrm{mg}$ of $\mathrm{KBr}$.

Crystallinity index: X-ray diffraction was used to determine cellulose acetate crystallinity index, following the same conditions used for cellulose.

\section{Results and discussions}

\subsection{Cellulose characterization}

Mercerization process alters the fine structure and morphology of the fiber as well as the conformation of the cellulose chains, hence altering the strength and shine of the fiber and its adsorption properties. During this process, the material swells and the polysaccharide chains are rearranged; the amount of less ordered material in the fiber rises, while the crystalline part contracts. These changes result in higher adsorption, given that mercerization

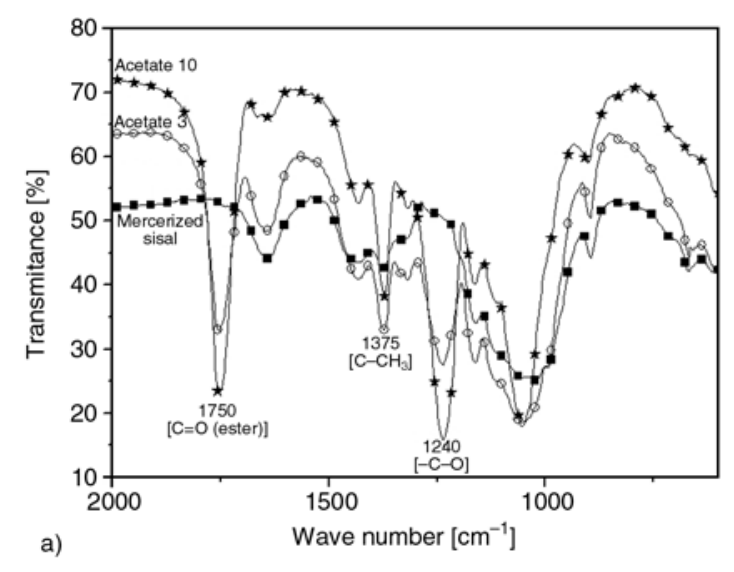

increases the specific surface area of the fiber, making the hydroxyl groups of cellulose macromolecules more accessible [8, 17] considered quite important for reactions occurring in heterogeneous medium, as for instance in the present work.

The degree of polymerization (DP) for untreated sisal was 760 and for mercerized sisal 670, indicating that the mercerization process possibly degraded the cellulose chains breaking some of the glycosidic bonds. For microcrystalline cellulose the DP was 126 [7].

The crystallinity index $\left(I_{c}\right)$ results were $77 \%$ for untreated sisal and $65 \%$ for mercerized sisal, confirming that mercerization causes reduction in cellulose crystallinity, as a diffusion consequence of $\mathrm{NaOH}$ into crystalline domains of cellulose, besides rearrangements that can take place in the crystal packing of the chains, as is well known $[8$, 18]. The $I_{c}$ for microcrystalline cellulose was $81 \%$.

\subsection{Cellulose acetates characterization}

The infrared spectra (Figure 2a) confirm the presence of acetyl groups, due to the absorption bands at $1750 \mathrm{~cm}^{-1}(\mathrm{vC}=\mathrm{O}), 1375 \mathrm{~cm}^{-1}\left(\mathrm{vC}-\mathrm{CH}_{3}\right)$ and $1235 \mathrm{~cm}^{-1}(\mathrm{vC}-\mathrm{O})$. The infrared spectra of the other acetates (figures not shown) have a similar profile to the present ones.

Figure $2 \mathrm{~b}$ shows the NMR spectrum of acetate 11 (Table 1), DS 3.0. The DS values of the cellulose acetates were calculated by means of the ratio between the area corresponding to the proton resonance of the glucose ring $(\delta \sim 2.90-5.10 \mathrm{ppm})$ and the corresponding resonance for the methyl protons of the acetate group $(\delta \sim 1.70-2.20 \mathrm{ppm})$ [15].

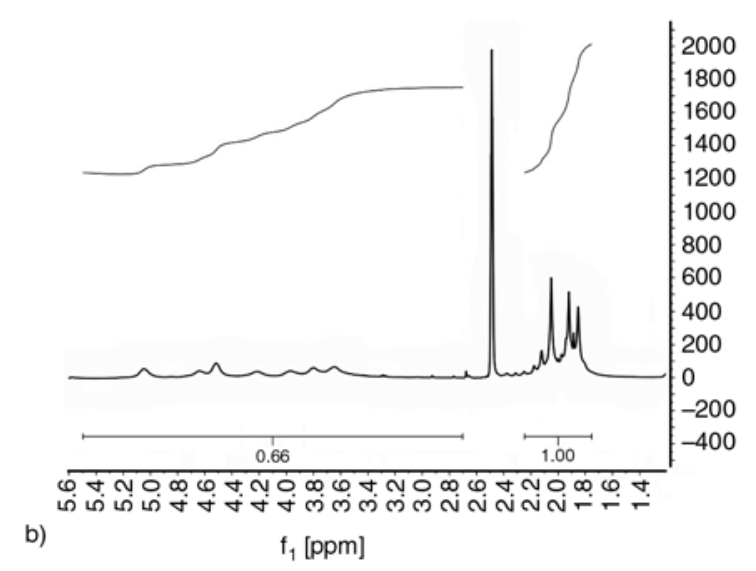

Figure 2. a) Infrared spectra of cellulose and cellulose acetates. Acetate 3 corresponds to DS $=0.5$ (reaction time $=$ $30 \mathrm{~min}$; sisal cellulose); acetate 10 corresponds to $\mathrm{DS}=2.7$ (reaction time $=30 \mathrm{~min}$; microcrystalline cellulose); b) ${ }^{1} \mathrm{H}$ NMR of microcrystalline cellulose acetate (sample 11, DS 3.0) 
Other NMR spectra (figures not shown) were similar to that of Figure $2 b$.

Table 1 presents the reaction time and the corresponding degrees of substitution and crystallinity index $\left(I_{c}\right)$ of the cellulose acetates.

The results (Table 1) show the catalytic effect of iodine, as when there is no catalyst, a very low DS was obtained (0.2, sample 1$)$ for the sisal cellulose, even with a reaction time of $360 \mathrm{~min}$. The presence of iodine led to approximately the same DS with a 10 min reaction (sample 2).

In heterogeneous medium, the accessibility of reagents to hydroxyl groups of the cellulose is dependent on its degree of crystallinity, among other factors. In general lines, the non-crystalline domains are more accessible to reactive agents making the reaction more feasible $[8,16]$. The results showed that although mercerized sisal cellulose has less crystalline domains than cellulose microcrystalline, for the same reaction time (compare acetates 2,3 , 4 with $9,11,12$ ), microcrystalline cellulose acetylation was considerably faster. This is quite probable due to the typical characteristics of the sisal cellulose, particularly because of its fibrous structure and higher average molar mass [2] that also can influence the accessibility of reagents to hydroxyl groups. Non-fibrous microcrystaline cellulose has organized domains of ca. $100 \mathrm{~nm}$, which is equivalent to the length of a chain of 200 anhydroglucose units, AGU. The penetration of a few reagent molecules into the suspended solid can be sufficient to perturb any long-range structural domains, therefore facilitating the continuity of the reaction. In this case, the

Table 1. Degree of substitution (DS) and crystallinity index $\left(I_{c}\right)$ of sisal (samples 1-8) and microcrystalline (samples 9-11) cellulose acetates

\begin{tabular}{|c|c|c|c|c|}
\hline $\begin{array}{l}\text { Acetate } \\
\text { sample }\end{array}$ & Cellulose & $\begin{array}{c}\text { Reaction time } \\
\text { [min] }\end{array}$ & DS & $\begin{array}{c}\mathbf{I}_{\mathbf{c}} \\
{[\%]}\end{array}$ \\
\hline $1^{\mathrm{a}}$ & \multirow{8}{*}{$\begin{array}{c}\text { Sisal } \\
\left(I_{C}=65 \%\right)\end{array}$} & 360 & 0.2 & 54 \\
\hline 2 & & 10 & 0.3 & 54 \\
\hline 3 & & 30 & 0.5 & 56 \\
\hline 4 & & 60 & 0.9 & 56 \\
\hline 5 & & 90 & 1.5 & 63 \\
\hline 6 & & 120 & 1.9 & 63 \\
\hline 7 & & 150 & 2.3 & 60 \\
\hline 8 & & 180 & 2.8 & 58 \\
\hline 9 & \multirow{3}{*}{$\begin{array}{l}\text { Microcrystalline } \\
\qquad\left(I_{c}=77 \%\right)\end{array}$} & 10 & 2.5 & 73 \\
\hline 10 & & 30 & 2.7 & 68 \\
\hline 11 & & 60 & 3.0 & 54 \\
\hline
\end{tabular}

a Iodine was not used in this acetylation reaction
DP value plays a more important role than crystallinity. By contrast, fibrous celluloses, as sisal, have high DP and possess organized domains whose length may reach $400 \mathrm{~nm}$. Such celluloses' long chains may be localized either in the non-crystalline or crystalline domains. When compared to microcrystalline cellulose, the destruction of this long-range order is more difficult, this in turn influences the reagents' accessibilities [5,7].

Thus, for microcrystalline cellulose, a degree of substitution close to 3.0 is already obtained in a 10 min reaction, while for sisal cellulose the same DS was obtained after $180 \mathrm{~min}$ (Table 1). Summarizing, these results show that, in general terms, the lower molar mass of microcrystalline cellulose allied to the fact that this cellulose is not fibered like sisal cellulose, did in fact assist its acetylation. Moreover, it can be considered difficult to eliminate all the hemicellulose in the lignocellulosic fibers, such as wood and sisal, used in the present work. The probable presence of this polysaccharide in sisal cellulose, in spite of the small fraction and considering that the previous mercerization eliminates hemicelluloses, could also exert some influence on the process.

Then, the excellent proposal made by Biswas and collaborators $[11,12]$ which considers that cellulose acetates with high degrees of substitution can be obtained within short periods of time under iodine catalysis, probably applies easily for notfibered and low molar mass celluloses. Celluloses from different origins must be studied individually, as for sisal cellulose, studied in the present work. At any rate, iodine proved to be an excellent catalyst for sisal cellulose, as already found by Biswas $[11,12]$ for other cellulose.

Under the reaction conditions used in the present work, the obtained degree of substitution (DS) for cellulose acetates is linearly correlated with the reaction time (Figure 3), which enables preparing acetates with desired DS using the straight line obtained.

The results show that for sisal cellulose, there is no direct correlation between the degree of substitution (DS) of cellulose acetates and crystallinity index $\left(I_{c}\right.$, Table 1). However, for all reactions, $I_{c}$ diminished compared to sisal cellulose, in all likelihood due to the higher volume of the acetyl group linked to oxygen, when compared to the hydrogen of hydroxyl groups, which can make difficult the 


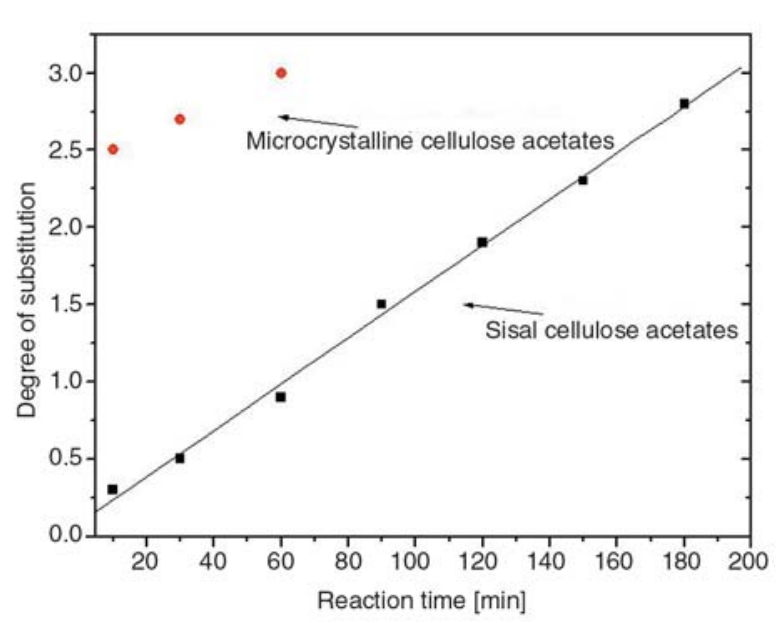

Figure 3. Degree of substitution versus reaction time for sisal and microcrystalline cellulose acetates

packing of chains. For microcrystalline cellulose acetates, the $I_{c}$ decreases as DS increases (Table 1), probably because acetyl groups were introduced more homogeneously along the short chains, when compared to sisal cellulose, leading then to a certain correlation between the DS values and $I_{c}$. However, the interval of DS is too short (2.5-3.0) for definitive conclusions on this subject.

\section{Conclusions}

The results demonstrate that iodine is an excellent catalyst for the reaction, since in its absence, the degree of acetylation of sisal cellulose is very low, even considering a larger reaction time, when compared to those obtained in the presence of iodine. The reaction is facilitated when lower molar mass and non-fibrous cellulose (microcrystalline) is used, probably due to the easier access of the reagent to the chains. Sisal cellulose acetates were prepared with varying reaction times, leading to products with degrees of substitution within a wide interval (DS between 0.3 and 2.8). Moreover, a linear correlation was observed between DS and reaction time, thus, it is possible to select the necessary conditions to synthesize sisal cellulose acetates with the targeted DS, which is an important finding, considering that cellulose acetates can have different applications, depending on their degree of acetylation.

\section{Acknowledgements}

E. F. is grateful to CNPq (National Council of Research, Brazil) for the research productivity fellowship and for financial support, and to FAPESP (The State of São Paulo Research Foundation, Brazil) for financial support.

\section{References}

[1] Ass B. A. P., Frollini E.: Aggregation of cellulose during dissolution and acetylation in $N, N$-dimethylacetamide/lithium chloride: An introductory study. Anais da Associação Brasileira de Química, 50, 76-82 (2001).

[2] Ciacco G. T., Ass B. A. P., Ramos L. A., Frollini E.: Acetylation of cellulose under homogeneous reaction conditions. in: 'Natural Polymers and Composites.' (eds.: Mattoso L. H. C., Leão A. L., Frollini E.) EMBRAPA, UNESP, USP, São Paulo, 139-145 (2000).

[3] Marson G. A., El Seoud O. A.: A novel, efficient procedure for acylation of cellulose under homogeneous solution conditions. Journal of Applied Polymer Science, 74, 1355-1360 (1999).

[4] El Seoud O. A., Marson G. A., Ciacco G. T., Frollini E.: An efficient, one-pot acylation of cellulose under homogeneous reaction conditions. Macromolecular Chemistry and Physics, 201, 882-889 (2000).

[5] Zhang M. Q.: Polymeric materials from natural resources - Emerging as the times require. Express Polymer Letters, 1, 406-406 (2007).

[6] El Seoud O. A., Regiani A. M., Frollini E.: Derivatization of cellulose in homogeneous conditions: A brief review. in: 'Natural Polymers and Agrofibers Based Composites' (eds.: Frollini E., Leão A. L., Mattoso L. H. C.) EMBRAPA, UNESP, USP, São Paulo, 73-89 (2000).

[7] Ass B. A. P., Ciacco G. T., Frollini E.: Cellulose acetates from linters and sisal: Correlation between synthesis conditions in $\mathrm{DMAc} / \mathrm{LiCl}$ and product properties. Bioresource Technology, 96, 1696-1702 (2005).

[8] Ramos L. A., Assaf M. J., El Seoud A. O., Frollini E.: Influence of the supramolecular structure and physicochemical properties of cellulose on its dissolution in a lithium chloride/ $N, N$-dimethylacetamide solvent system. Biomacromolecules, 6, 2638-2647 (2005).

[9] Ass B. A. P., Belgacem M. N., Frollini E.: Mercerized linters cellulose: Characterization and acetylation in $N, N$-dimethylacetamide/lithium chloride. Carbohydrate Polymers, 63, 19-29 (2006).

[10] Castellan A., Ruggiero R., Frollini E., Ramos L. A., Chirat C.: Studies on fluorescence of cellulosics. Holzforschung, 61, 504-508 (2007).

[11] Biswas A., Shogren R. L., Willet J. L.: Solvent-free process to esterify polysaccharides. Biomacromolecules, 6, 1843-1845 (2005). 
[12] Biswas A., Selling G., Appell M., Woods K. K., Willett J. L., Buchanan C. M.: Iodine catalyzed esterification of cellulose using reduced levels of solvent. Carbohydrate Polymers, 68, 555-560 (2007).

[13] Mukhopadhyay B., Ravindranathan K. P., Russel D. A., Field R. A.: Streamlined synthesis of per- $O$-acetylated sugars. Glycosyl iodides, or thioglycosides from unprotected reducing sugars. Journal of Organic Chemistry, 69, 7758-7760 (2004).

[14] Phukan P.: Iodine as an extremely powerful catalyst for the acetylation of alcohols under solvent-free conditions. Tetrahedron Letters, 45, 4785-4787 (2004).

[15] Buschle-Diller G., Zeronian S. H.: Enhancing the reactivity and strength of cotton fibers. Journal of Applied Polymer Science, 45, 967-979 (1992).
[16] Edgar K. J., Arnold K. M., Blount W. W., Lawniczak J. E., Lowmann D. W.: Synthesis and properties of cellulose acetoacetates. Macromolecules, 28, 4122 4128 (1995).

[17] Pušic T., Grancaric A. M., Soljacic I., Ribitsch V.: The effect of mercerisation on the electrokinetic potential of cotton. Journal of the Society of Dyers and Colourists, 115, 121-124 (1999).

[18] Nishiyama Y., Langan P., Chanzy H.: Crystal structure and hydrogen-bonding system in cellulose I from synchrotron X-ray and neutron fiber diffraction. Journal of American Chemical Society, 124, 9074-9082 (2002). 